\title{
Cold South Dakota winters...and dry eyes
}

\section{Editorial}

I was born and raised in South Dakota and I now live here again in my adult life. In my 20 years of practice, I have had the chance to visit many different parts of our great country. Yet with all the many great and amazing places to live, I choose to come back to my home state. Winters are cold and long. Most days from December to March, it's dirty, sloppy, dry, cold, irritating, and miserable. It also sounds like some of the ways my patients complain or describe their dry eye symptoms. I chose to live in this state and the environment that it is. Patients sometime choose to follow through with my treatment recommendations regarding their dry eye disease and some unfortunately do not. The living conditions in South Dakota will never change, as is it with dry eye disease. Both are chronic conditions. In both situations though, one needs to accept the conditions and manage them in order to make living conditions tolerable.

The way I treat dry eye syndrome today has changed from when I first started practicing, but so has the understanding of what dry eye disease is all about, the diagnostics available, and the products and treatments for patients to utilize. I was taught that having a dry eye was that patients had an issue with decrease tear production. They needed to use artificial tears or lubricating drops and/or think about adding tear duct plugs. Although these are very useful and acceptable ways to manage dry eyes, there is a fundamental shift in understanding what dry eye disease is in the first place. Dry eye disease, yes disease, is a chronic condition of either having a decrease aqueous production or an increase in evaporation. In fact, $86 \%$ of dry eye conditions are actually evaporative in nature. Pure aqueous deficiency is only about $10 \%$ of the cases. Many of the root causes of evaporative dry eye stem from patients having meibomian gland deficiency caused by not blinking appropriately. This causes stagnation in the glands, causing the meibom (oil) in the glands to harden, later to further leading to gland atrophy.

To truly be able to understand what type of dry eye condition a patient might have, a complete (and consistent) dry eye evaluation needs to be conducted. This begins by using a standardized patient questionnaire to understand the subjective symptoms and to be able to track those symptoms over time. I use the SPEED (Standardized Patient Evaluation of Eye Dryness) questionnaire, including asking specifically if the patient is having fluctuating vision. That is a major contributor to having meibomian gland disease. We then have our technicians use the Lipiview II (Tear Science) to ascertain the patient's meibomian gland function. This system allows us to visualize the meibomian glands themselves as well as looking at their lipid layer thickness and blink rate analysis. We then have the technicians check tear osmolarity using the TearLab system (Tear Science), inflammation with InflammaDry, and perform an anesthetized Schirmer's test.

We recommend patients not wear any contact lenses or use any eye drops two hours prior to coming in for their appointment. This allows consistency and accuracy in our testing and we are able to better track how our treatment plans are working. As the optometrist that next comes in to meet the patient, I check corneal staining with sodium fluorescein, conjunctival staining with lissamine green, and do manual expression of the meibomian glands. You need to remember to only

\author{
Volume 4 Issue I - 2016 \\ Jason Schmit
Department of Executive Operations Management Vance
Thompson Vision, USA
}

Correspondence: Jason Schmit, OD Oculoplastic Corneal Cataract and Refractive Surgery Specialist, Department of Executive Operations Management Vance Thompson vision, Artisian 57 Sioux falls SD, USA, Tel 605-95I-2135, Email jason.schmit@vancethompsonvision.com

Received: January 26, 2016 | Published: January 27, 2016

press on the glands with the same pressure that is exhibited with a blink. The manual gland depressor (MGD by Tear Science) is a good device to use to simulate that force.

As the dry eye specialist in our practice, I meet with the patients and review the data thus far. Having the doctor educate patients on the testing results and explaining (with visuals) the type of dry eye condition they might have is instrumental in the process.

Many of the patients I see in a specialty referral clinic like ours may not have had advanced testing or an explanation of their condition discussed with them like we have established before. The process of diagnosing, educating, and providing a customized treatment plan specific to their condition is what I believe sets us apart from most practices. Analyzing all the data regarding dry eye disease is analogous to analyzing all the data to diagnose glaucoma. For example, you look at IOP, CD ratios, pachymetry, NFL analysis, visual fields, gonioscopy, and hysteresis. You make an educated decision on how to treat each patient based on the findings. You do the exact same when managing dry eye disease too.

I like to start by recommending artificial tears with sodium hyaluronate. It's a great lubricant and helps control inflammation. Blink (Bausch and Lomb) is the brand I use most. In more severe cases, I use a compounding pharmacy to make $100 \%$ Healon into non-preserved eye drops that work well too. For pure aqueous deficiency, I next recommend utilizing tear duct plugs, if there isn't any inflammation present as determined by the InflammaDry test. I have access to about different 4-5 styles to choose from but the one I use most is Ultraplug. It comes in a variety of sizes, easy to insert, and actually is cost effective. I also prescribe Restasis (Allergan) to be used twice daily, especially if there is some inflammation and/or a decrease in the anesthetized Schirmer's. If I'm concerned that the cause of a patient's dry eye condition might be stemming from an autoimmune disease like Sjogren's syndrome, the Sjo test (Bausch and Lomb) has been an invaluable adds to the practice. I probably diagnose 5-10 new cases a month and then refer on to rheumatology.

Again since most of my dry eye patients are evaporative in nature, much of what I recommend for treatments focuses on that being the root cause. Warming compress and lid message are using my first 
go to recommendation. We have a retail area in our practice where all the products I'll mention next are sold. This helps with patient compliance and convenience and makes getting treatments initiated a much faster process. The warming mask we retail is the Fire and Ice mask. It has a removable fabric overlay that is washable and can kept in the freezer for a cold mask, or micro waved for 10-15 seconds for a warming mask that lasts about 10 minutes. Since the meibomian glands are actually closer to the back of the eyelids, having a treatment like Lipiflow (Tear Science) has been a much better way to go. Over the last year we have completed about 75 treatments on patients and have found that system to be very effective. I tell patients that they will still have this chronic condition and will need to go through the treatment again. They will have diminished symptoms, and by having a treatment, it will allow them to possibly use less at home therapies so they can get on with their lives. Regarding at home warming masks, the vasculature in the skin and tarsal plate within the eyelid does prevent much of the effectiveness of the heat to diminish. It the best that's available though and I definitely have patients stay away from other home remedies that include food items (hot potato, hard boiled eggs, rice packs, etc.) Keep those items for your mouth and not your eyes!

If there is an inflammatory component to their eye lids or a rosacea/ocular rosacea present, I use Omega 3 fish oil. Most of our diets in the US consist of corn fed beef and processed foods, which are high in Omega 6 fatty acids. These actually promote inflammation and the ratio of Omega 3's to Omega 6's needs to be brought back into balance. PRN, Nordic Naturals, or Fortifeye are products I like the best because they are the pure triglyceride forms of the product, not the alcohol forms like most Omega 3's on the market. I usually recommend taking at least $2000 \mathrm{mg}$ per day. I also recommend using Azasite daily by having patients rub the eye drop into the lid margin. Oral doxycycline/minocycline at $50 \mathrm{mg} / \mathrm{bid}$ is also effective in helping control inflammation. Remember though that these prescription medications are to help manage the condition and symptoms of inflammation, but they do not address root cause of the disease. Another great treatment we have available is BBL (Sciton) therapy to the eyelids. Utilizing light therapy has been proven to control inflammation within the skin by targeting superficial blood vessels. Heat is absorbed in the vessels and coagulates the cells, leading to a thrombosis of the blood vessels. It has in impact on the bacterial flora on the skin and eyelids, as well as increasing the skin temperature, all of which have a beneficial effect on the meibomian glands.

For more severe cases of dry eye disease in which there is a persistent corneal keratitis present, I prescribe a topical steroid
(Lotemax - Bausch and Lomb) to be used four times a day for 1 week, then twice a day for 1-2 weeks. I also prescribe Autologous Serum Tears (AST) to be used every 1-2 hours. The compounding pharmacy we use can make these for about $\$ 350$ for a 2-3 month's supply for the patient. Our aesthetic skin clinic is also using PRP for CIT, so if your clinic prescribes a lot of AST, I might make sense to bring this in house and have your own system available. Amniotic membranes have also been very effective in treating severe keratitis conditions. I use the ProKera slim and have the patient keep this on for 1 week. Remind them to keep their eye taped at night as this can possibly fall out. Since we are an ophthalmology surgical practice and I also specialize in oculoplastics, taking into account the eyelid anatomy and positioning can definitely be a factor in many of the eye symptoms patients' experience. Water eyes can stem from blocked tear ducts, laxity or lagophthalmos, conjunctivochalasis, pinguecula, pterygia, and the like. Blepharitis is very common. In fact I assume most patients have blepharitis until proven otherwise. I like to use Avenova (NovaBay) to treat lid margin disease. It contains pure hypochlorous acid which can remove microorganisms and debris from the lids and lashes. It's also great eradicating demodex and helps fight inflammation.

This obviously is just a snapshot of what can and needs to go into diagnosing and managing dry eye patients. The science around this practice of medicine has definitely changed over the years and has really changed my thinking of how to help these patients. I know in my travels, there is parts of the country that have drier conditions way more than South Dakota. Colorado with the elevation or Arizona being a desert were two states that I had visited with many more advanced dry eye patients. As I look outside my window in Sioux Falls, it's snowing now and I'm wishing that I was down in Arizona. But I stay warm and dress in layers. I wear my hat and gloves, and make sure my all-wheel drive car is in top working condition. These are the things you do in South Dakota to survive the winter. Likewise, there are things that we can also do to manage dry eye conditions so patients don't have to dwell on the symptoms of having dirty, sloppy, dry, cold, irritating, and miserable eyes.

\section{Acknowledgments}

None.

\section{Conflicts of interest}

The author declares there is no conflict of interest. 